\title{
Mutations at amino-acid 482 in the ABCG2 gene affect substrate and antagonist specificity
}

\author{
RW Robey",', Y Honjo', K Morisaki', TA Nadjem', S Runge', M Risbood', MS Poruchynsky' and SE Bates' \\ 'National Institutes of Health, Center for Cancer Research, Cancer Therapeutics Branch, Bethesda, MD 20892, USA
}

\begin{abstract}
Recent studies have shown that mutations at amino-acid 482 in the ABCG2 gene affect the substrate specificity of the protein. To delineate the effects of these mutations clearly, human embryonic kidney cells (HEK-293) were stably transfected with wild-type $482 R$ or mutant $482 \mathrm{G}$ and $482 \mathrm{~T} \mathrm{ABCG2}$. By flow cytometry, mitoxantrone, BODIPY-prazosin, and Hoechst 33342 were found to be substrates of all ABCG2 proteins, while rhodamine 123, daunorubicin, and LysoTracker Green were transported only by mutant ABCG2. In cytotoxicity assays, all ABCG2 proteins conferred high levels of resistance to mitoxantrone, $\mathrm{SN}-38$, and topotecan, while mutant $A B C G 2$ also exhibited a gain of function for mitoxantrone as they conferred a four-fold greater resistance compared to wild type. Cells transfected with mutant ABCG2 were I3- to 7 I - fold resistant to the P-glycoprotein substrates doxorubicin, daunorubicin, epirubicin, bisantrene, and rhodamine 123 compared to cells transfected with wild-type ABCG2, which were only three- to four-fold resistant to these compounds. ABCG2 did not confer appreciable resistance to etoposide, taxol or the histone deacetylase inhibitor depsipeptide. None of the transfected cell lines demonstrated resistance to flavopiridol despite our previous observation that ABCG2-overexpressing cell lines are cross-resistant to the drug. Recently reported inhibitors of ABCG2 were evaluated and $50 \mu \mathrm{M}$ novobiocin was found to reverse wild-type ABCG2 completely, but only reverse mutant $A B C G 2$ partially. The studies presented here serve to underscore the importance of amino-acid 482 in defining the substrate specificity of the ABCG2 protein and raise the possibility that amino-acid 482 mutations in human cancers could affect the clinical application of antagonists for ABCG2.
\end{abstract}

British Journal of Cancer (2003) 89, 197I - 1978. doi:10.1038/sj.bjc.660I370 www.bjcancer.com

(c) 2003 Cancer Research UK

Keywords: drug-resistance; BCRP/MXR/ABCP/ABCG2; mitoxantrone; flow cytometry; mutation

ABCG2 is an ATP-binding cassette half-transporter (Allikmets et al, 1998; Doyle et al, 1998; Miyake et al, 1999) that has been shown to confer resistance to a variety of chemotherapeutic agents including mitoxantrone (Brangi et al, 1999; Litman et al, 2000); the camptothecins topotecan (Maliepaard et al, 1999; Yang et al, 2000) and SN-38 (Kawabata et al, 2001); doxorubicin (Chen et al, 1990); and flavopiridol (Robey et al, 2001b). Cells overexpressing wild-type ABCG2 with an arginine at amino-acid 482 have been shown by flow cytometric analysis to transport mitoxantrone, while those overexpressing ABCG2 with a threonine or glycine at position 482 (R482G, R482T) transported mitoxantrone and also exhibited a gain in function with the transport of rhodamine 123 and daunorubicin (Honjo et al, 2001). In Cytotoxicity assays, cells overexpressing any of the ABCG2 proteins are resistant to mitoxantrone, topotecan, and SN-38, while cells overexpressing mutant ABCG2 are additionally resistant to the anthracyclines. Although these phenotypic differences have been well described in selected cell lines, careful studies in transfected cells were needed to exclude bias due to other mechanisms of resistance in selected cell lines.

\footnotetext{
* Correspondence: RW Robey, Center for Cancer Research, 9000 Rockville Pike, Bldg 10 Rm I2Cl03, Bethesda, MD 20892, USA; E-mail: robeyr@mail.nih.gov

Received 21 January 2003; revised 20 June 2003; accepted II September 2003
}

A similar situation was recently described for the mouse homologue, Abcg2. Allen et al (1999) reported overexpression of Abcg2 in mouse fibroblast cell lines lacking functional Mdrla, Mdr1b, and Mrp1, which were selected in mitoxantrone, doxorubicin, or topotecan. Greater doxorubicin resistance was observed in the cells selected in doxorubicin compared to those selected in mitoxantrone or topotecan. Upon sequencing Abcg2 in these cell lines and two other fibroblast cell lines selected in doxorubicin, it was noted that the arginine at position 482 in wild-type Abcg 2 was mutated to a methionine or serine. Expression of the mutant Abcg2 proteins conferred greater resistance to the anthracyclines as well as the ability to transport rhodamine 123 (Allen et al, 2002). Allen suggested that the 482 residue represented a hot spot for mutation in ABCG2. Subsequent to these studies, an arginine to methionine mutation was also described in a selected human cell line (Wang et al, 2003).

To characterize precisely the cross-resistance profile conferred by the wild-type and mutant ABCG2 proteins, we transfected human embryonic kidney (HEK-293) cells with 482R, 482T, or 482G ABCG2. In transfected cell lines expressing comparable levels of ABCG2, we tested the ability of the transfectants to transport fluorescent compounds reported to be substrates of the halftransporter, and performed killing curves with chemotherapeutic agents reported to be effluxed by ABCG2 to determine the crossresistance profile conferred by each of the ABCG2 proteins.

The interactions of the recently reported ABCG2 antagonists novobiocin (Doyle et al, 2002; Shiozawa et al, 2002), $\beta$-estradiol, and estrone (Imai et al, 2002) with wild-type and mutant ABCG2 
were also examined. The results presented here demonstrate amino-acid 482 to be significant in determining the effectiveness of ABCG2 antagonists as well as the ability to transport selected antineoplastic drugs.

\section{MATERIALS AND METHODS}

\section{Chemicals}

The fluorescent compounds LysoTracker Green DND-26 and BODIPY-prazosin were purchased from Molecular Probes (Eugene, OR, USA). Flavopiridol, topotecan, depsipeptide, and epirubicin were obtained from the National Cancer Institute Drug Screen. Mitoxantrone, daunorubicin, doxorubicin, Hoechst 33342, rhodamine 123, novobiocin, estrone, and $\beta$-estradiol were purchased from Sigma Chemical Co. (St Louis, MO, USA). Bisantrene was a gift from Dr Lee Greenberger (Wyeth-Ayerst). Fumitremorgin C (FTC) was synthesised by Thomas McCloud, Developmental Therapeutics Program, Natural Products Extraction Laboratory, National Institutes of Health (Bethesda, MD, USA).

\section{Establishment of stable transfectants}

HEK-293 cells were transfected with either empty pcDNA3 vector (Invitrogen, Carlsbad, CA, USA) or pcDNA3 vector containing fulllength ABCG2 coding either an arginine, threonine or glycine for amino-acid 482. Expression of ABCG2 in the transfectants was enforced by selection in G418 (Invitrogen, Carlsbad, CA, USA). Stable transfectants were maintained in Eagle's minimum essential medium (ATCC, Manassas, VA, USA) supplemented with $10 \%$ FCS, penicillin, and streptomycin with G418 at a concentration of $2 \mathrm{mg} \mathrm{ml}^{-1}$. Clones were preliminarily screened for ABCG2 expression by examining the ability of the cells to efflux BODIPYprazosin in a flow cytometry-based assay. The ABCG2 sequence was subsequently verified in the clones examined here.

\section{RNA isolation and Northern blot analysis}

RNA was extracted from cells using RNA STAT-60 (Tel-Test Inc., Friendswood, TX, USA) according to the manufacturer's instructions. Northern blot analysis was performed using a riboprobe generated from the first $662 \mathrm{bp}$ of ABCG2 subcloned into a pCRIITOPO vector (Invitrogen, Carlsbad, CA, USA).

\section{Western blot analysis}

Microsomal membrane fractions $(30 \mu \mathrm{g})$ were subjected to electrophoresis and transferred to nitrocellulose membranes as previously described (Robey et al, 2001b). Blots were probed with the monoclonal anti-BCRP antibody BXP-21 (Maliepaard et al, 2001) (Kamiya Biomedical, Seattle WA, USA) as previously described (Litman et al, 2000).

\section{Flow cytometry}

Flow cytometry assays were performed as previously described (Robey et al, 2001a). Briefly, cells were trypsinised, resuspended in complete media (phenol red-free IMEM with $10 \%$ fetal calf serum) containing $20 \mu \mathrm{m}$ mitoxantrone, $5 \mu \mathrm{g} \mathrm{ml}^{-1}$ daunorubicin, $250 \mathrm{nM}$ BODIPY-prazosin, $0.5 \mu \mathrm{g} \mathrm{ml}^{-1}$ rhodamine 123, $250 \mathrm{~nm}$ LysoTracker Green DND-26, or $10 \mu \mathrm{M}$ Hoechst 33342 with or without $10 \mu \mathrm{M}$ of the ABCG2 blocker, FTC, and incubated for $30 \mathrm{~min}$ at $37^{\circ} \mathrm{C}$ in $5 \%$ $\mathrm{CO}_{2}$. FTC has been reported to be a specific blocker of ABCG2 (Rabindran et al, 1998, Rabindran et al, 2000), and we assumed $10 \mu \mathrm{M}$ FTC afforded complete prevention of ABCG2-mediated efflux based on previous results. In studies with reported ACBG2 antagonists, cells were incubated with $250 \mathrm{~nm}$ BODIPY-prazosin alone or with various concentrations of the inhibitors. Cells were then washed once in cold complete medium and then incubated for $1 \mathrm{~h}$ at $37^{\circ} \mathrm{C}$ in substrate-free media continuing with or without $10 \mu \mathrm{M}$ FTC or the described concentrations of the other ABCG2 inhibitors to generate the efflux and FTC/efflux histograms (or inhibitor/efflux histograms), respectively. Subsequently, cells were washed twice with cold DPBS and placed on ice in the dark until analysed. FTC/efflux-efflux values, calculated as the difference in mean channel numbers between the FTC/efflux and efflux peaks, were generated for each cell line with each fluorescent substrate. Cells were analysed either on a FACSort flow cytometer equipped with both a $488 \mathrm{~nm}$ argon laser and a $635 \mathrm{~nm}$ red diode laser or a FACSVantage flow cytometer equipped with a $360 \mathrm{nM}$ UV laser to detect Hoechst 33342 fluorescence. For all samples, at least 10000 events were collected. Debris was eliminated by gating on forward $v s$ side scatter and dead cells were excluded based on propidium iodide staining.

For studies with the anti-ABCG2 antibody, 5D3, cells were trypsinised and resuspended in DPBS with $2 \%$ BSA to which was added phycoerythrin-conjugated 5D3 (eBioscience, San Diego, CA, USA) or phycoerythrin-conjugated mouse IgG. The cells were incubated with antibody for $30 \mathrm{~min}$ at room temperature, washed twice with DPBS and kept in the dark until analysed.

\section{Cytotoxicity assays}

The cytotoxocity assays performed were based on the previously described sulphorhodamine B assay (Skehan et al, 1990). Cells were plated in flat-bottom 96-well plates at a density of 2000 cells per well and allowed to attach for $24 \mathrm{~h}$ at $37^{\circ} \mathrm{C}$ in $5 \% \mathrm{CO}_{2}$. Chemotherapeutic agents at various concentrations were added to the cells and the plates were allowed to incubate for $96 \mathrm{~h}$ at $37^{\circ} \mathrm{C}$ in $5 \% \mathrm{CO}_{2}$. Subsequently, cells were fixed in $12.5 \%$ trichloroacetic acid and then stained with sulphorhodamine $\mathrm{B}$ solution $(0.4 \%$ sulphorhodamine $\mathrm{B} \mathrm{wv} \mathrm{w}^{-1}$ in $1 \%$ acetic acid). Optical densities were read on a Bio-Rad plate reader at an absorbance of $540 \mathrm{~nm}$. Each concentration was tested in triplicate and controls were performed in replicates of eight.

\section{RESULTS}

\section{Expression of ABCG2 in HEK-293 cells}

HEK-293 cells were transfected with the ABCG2 gene and screened based on BODIPY-prazosin transport (data not shown). From the positive clones obtained, two clones transfected with each ABCG2 gene (482G-1, 482G-2, 482R-2, 482R-5, 482T-7, 482T-10) and one clone transfected with empty vector (pcDNA3-10) were selected and RNA was extracted. Northern blotting was performed using $20 \mu \mathrm{g}$ total RNA. As shown in Figure 1A, all clones transfected with ABCG2 have enforced expression of the ABCG2 gene at the RNA level; in the cell line transfected with empty vector, no ABCG2 RNA was detected. Western blotting was performed on $30 \mu \mathrm{g}$ membrane protein isolated from the seven cell lines and comparable protein expression was observed (Figure 1B). Serial dilutions of protein from the transfectants used in cytotoxicity assays revealed about a two-fold higher level in wild-type-expressing cells (482R-2) than the mutant-expressing cells (482G-1, 482T-10) as seen in Figure 1C. The seven cell lines shown in Figure $1 \mathrm{~A}$ were then incubated with an anti-ABCG2 monoclonal antibody, clone 5D3 (Zhou et al, 2001), to measure the expression of ABCG2 on the cell surface by flow cytometry. All stably transfected cell lines demonstrated comparable expression of ABCG2 on the cell surface, with the empty vector transfected cells expressing no ABCG2 (Figure 1D); the difference in channel numbers between cells incubated with negative control antibody and the anti-ABCG2 antibody 5D3 are given in Table 1. Despite higher ABCG2 expression at the RNA 
A
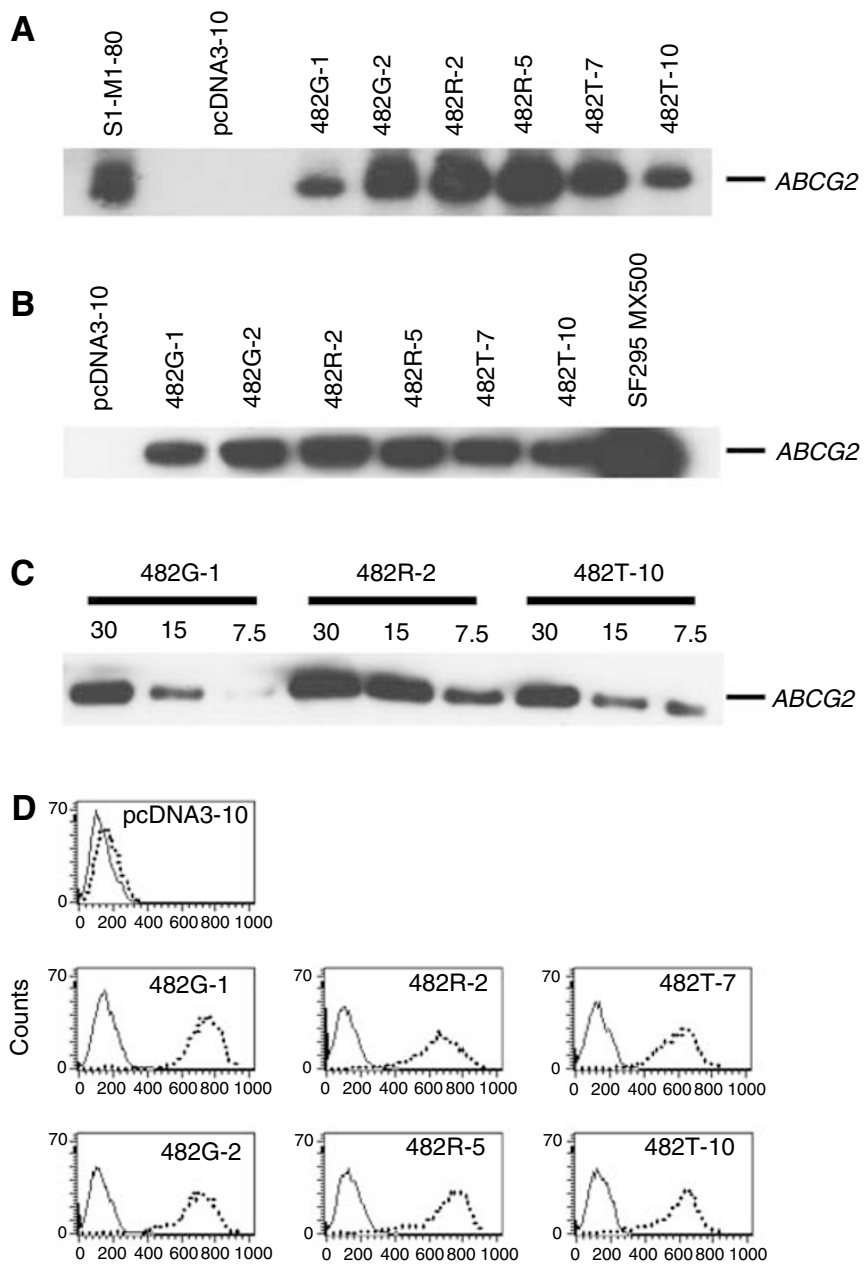

Phycoerythrin fluorescence

Figure I Expression of ABCG2 in HEK-293 cells transfected with wildtype and mutant ABCG2. (A) Northern blot analysis was performed on $20 \mu \mathrm{g}$ RNA extracted from six cell lines transfected with ABCG2 (482G-I, 482G-2, 482R-2, 482R-5, 482T-7, 482T-10) and one cell line transfected with empty vector (pcDNA3-10) and probed with a riboprobe generated from the first 662 bp of ABCG2. (B) Immunoblot analysis for ABCG2 using the anti-ABCG2 antibody BXP-2I was performed on membrane protein $(30 \mu \mathrm{g})$ from the seven transfected cell lines. (C) Immunoblot analysis for ABCG2 with serial dilutions of membranes isolated from same transfected clones (482G-I, 482R-2, 482T-10) subsequently used in cytotoxicity assays. (D) The seven transfected cell lines were incubated for 30 min with either phycoerythrin-labelled negative control antibody (dotted line) or phycoerythrin-labelled anti-ABCG2 antibody (dashed line) and then analysed on a flow cytometer. Representative histograms are shown.

level in the two 482R clones, similar levels of ABCG2 protein were found in all of the transfectants. The sequence of the transfected ABCG2 was confirmed in the six positive clones.

\section{Transport of fluorescent substrates by ABCG2-transfected} HEK-293 cells

The amino acid at position 482 has been found to be predictive for the ability of ABCG2 to transport fluorescent substrates (Honjo et al, 2001). Representative histograms for rhodamine 123, daunorubicin, mitoxantrone, BODIPY-prazosin, and LysoTracker Green are shown in Figure 2. Here the dotted lines represented the accumulation of the substrate in the presence of the ABCG2 inhibitor fumitremorgin $C$, while the solid line represents the accumulation without inhibitor. Reduced accumulation that is increased by FTC is present to varying degrees in the $482 \mathrm{G}$ and $482 \mathrm{~T}$ mutants for all of the substrates. In contrast, those vectors carrying the wild-type (482R) ABCG2 showed evidence of transport only of mitoxantrone and BODIPY-prazosin. FTC/ efflux-efflux values for BODIPY-prazosin, rhodamine 123, daunorubicin, LysoTracker Green DND-26, and mitoxantrone were calculated for each cell line. We have previously shown that FTCinhibitable mitoxantrone or BODIPY-prazosin efflux (FTC/effluxefflux value) is proportional to the expression of ABCG2 in cell lines (Robey et al, 2001a). The results are summarised in Table 1; negative FTC/efflux-efflux values were given the value of zero. FTC/efflux-efflux values for mitoxantrone and BODIPY-prazosin were comparable among the transfected cell lines. However, efflux of rhodamine 123, LysoTracker Green and daunorubicin is only observed in HEK-293 cells transfected with mutant $482 \mathrm{G}$ or $482 \mathrm{~T}$ ABCG2. As the dye Hoechst 33342 has recently been shown to be a substrate of ABCG2 (Zhou et al, 2001; Kim et al, 2002; Scharenberg et al, 2002), the ability of the three ABCG2 proteins to transport the compound was evaluated and confirmed for all three proteins. None of the fluorescent compounds was transported by the cell line transfected with empty vector (data not shown).

\section{Sensitivity of HEK-293 cells transfected with wild-type or mutant ABCG2 to antineoplastic agents}

The 4-day cytotoxicity assays were performed on the empty vector transfected cells as well as the 482G-1, 482R-2, and $482 \mathrm{~T}-10$ transfected clones. The results are summarised in Table 2; representative curves for some of the compounds tested are shown in Figure 3. In agreement with previous reports, HEK-293 cells transfected with any of the ABCG2 genes exhibited high levels of resistance to mitoxantrone compared to empty vectortransfected cells; however, $\mathrm{IC}_{50}$ values for $482 \mathrm{G}-1$ and $482 \mathrm{~T}-10$, 543 and $457 \mathrm{~nm}$ respectively, were about four-fold higher than for the 482R-2 cells, $107 \mathrm{~nm}$. When cytotoxicity experiments were performed with mitoxantrone on another set of clones, 482G-2, 482R-5, and 482T-7, the HEK-293 cells expressing either of the mutant ABCG2 proteins (482G-2, 482T-7) had $\mathrm{IC}_{50}$ values that were again about four-fold higher than for cells expressing wildtype 482R-5 ABCG2 (data not shown). Both sets of experiments suggest that mitoxantrone is a better substrate for mutant ABCG2 than wild type. HEK-293 cells expressing any of the ABCG2 proteins demonstrated 21- to 34 -fold resistance to topotecan and 48- to 96 -fold resistance to SN-38. For either drug, the $\mathrm{IC}_{50}$ of the $482 \mathrm{R}$ wild-type clone was higher than that observed in the clones expressing mutant ABCG2. Cells transfected with wild-type ABCG2 showed little resistance to the anthracyclines doxorubicin and daunorubicin, as well as bisantrene, epirubicin, and rhodamine 123 , compared to mutant ABCG2. ABCG2 conferred low levels of resistance to etoposide, but did not confer obvious resistance to depsipeptide or paclitaxel. Surprisingly, none of the ABCG2 proteins conferred appreciable resistance to flavopiridol. This is in contrast to a previous finding showing resistance to flavopiridol in selected cell lines overexpressing ABCG2 and overexpression of ABCG2 in cell lines selected in flavopiridol (Robey et al, 2001b).

\section{Effect of amino-acid 482 mutations on antagonist efficacy}

As mutations in Pgp have been shown to affect the ability of Pgp antagonists to prevent Pgp-mediated efflux (Chen et al, 1997; Ma et al, 1997; Watanabe et al, 2000), we examined the impact of the mutations at amino-acid 482 on ABCG2 antagonists. ABCG2transfected cells were incubated for $30 \mathrm{~min}$ in $250 \mathrm{~nm}$ BODIPYprazosin alone, or with $50 \mu \mathrm{m}$ novobiocin, $100 \mu \mathrm{m}$ novobiocin, $100 \mu \mathrm{m} \beta$-estradiol, $100 \mu \mathrm{m}$ estrone, or $10 \mu \mathrm{m}$ FTC. Cells were then washed and incubated for $1 \mathrm{~h}$ in substrate-free media continuing without or with the desired blocker to generate the efflux or inhibitor/efflux histograms, respectively. The FTC/efflux histogram 
Table I Antibody labelling and FTC-inhibitable efflux of fluorescent compounds in ABCG2 transfected cell lines

\begin{tabular}{|c|c|c|c|c|c|c|c|}
\hline Cell line & $5 D 3^{a}$ & BODIPY-prazosin ${ }^{\text {b }}$ & Mitoxantrone $^{b}$ & LysoTracker & Daunorubicin $^{b}$ & Hoechst $33342^{b}$ & Rhodamine $123^{b}$ \\
\hline 482G-I & $531.0 \pm 42.6$ & $314.2 \pm 21.4$ & $183.3 \pm 16.6$ & $91.3 \pm 2.1$ & $117.9 \pm 1.2$ & $208.0 \pm 12.7$ & $241.9 \pm 18.9$ \\
\hline 482G-2 & $504.2 \pm 41.3$ & $294.7 \pm 26.1$ & $181.9 \pm 24.3$ & $77.7 \pm 10.0$ & $137.36 \pm 10.2$ & $234.8 \pm 8.2$ & $214.9 \pm 27.1$ \\
\hline 482R-2 & $474.7 \pm 51.6$ & $186.9 \pm 41.2$ & $120.6 \pm 16.5$ & $18.4 \pm 10.4$ & $4.4 \pm 0.4$ & $164.8 \pm 4.3$ & 0 \\
\hline $482 R-5$ & $557.2 \pm 16.4$ & $233.3 \pm 13.6$ & $162 \pm 9.8$ & $21.5 \pm 14.7$ & $12.4 \pm 0.6$ & $212.1 \pm 16.5$ & 0 \\
\hline $482 T-7$ & $443.5 \pm 30.1$ & $259.6 \pm 18.8$ & $137.3 \pm 25.8$ & $75.7 \pm 8.6$ & $128.98 \pm 14.6$ & $216.7 \pm 3.3$ & $238.2 \pm 13.2$ \\
\hline $482 \mathrm{~T}-10$ & $471.4 \pm 18.6$ & $223.3 \pm 34.9$ & $143.8 \pm 25.4$ & $85.39 \pm 16.2$ & $166.3 \pm 6.9$ & $199.6 \pm 5.1$ & $232.4 \pm 28.2$ \\
\hline
\end{tabular}

${ }^{a}$ The values calculated are the difference in mean channel numbers between the 5D3 antibody histogram and the negative control antibody histogram. ${ }^{b}$ Difference in mean channel number between the FTC/efflux histogram and the efflux histogram. Negative values were assigned the value of zero. Values obtained were from at least two independent experiments.
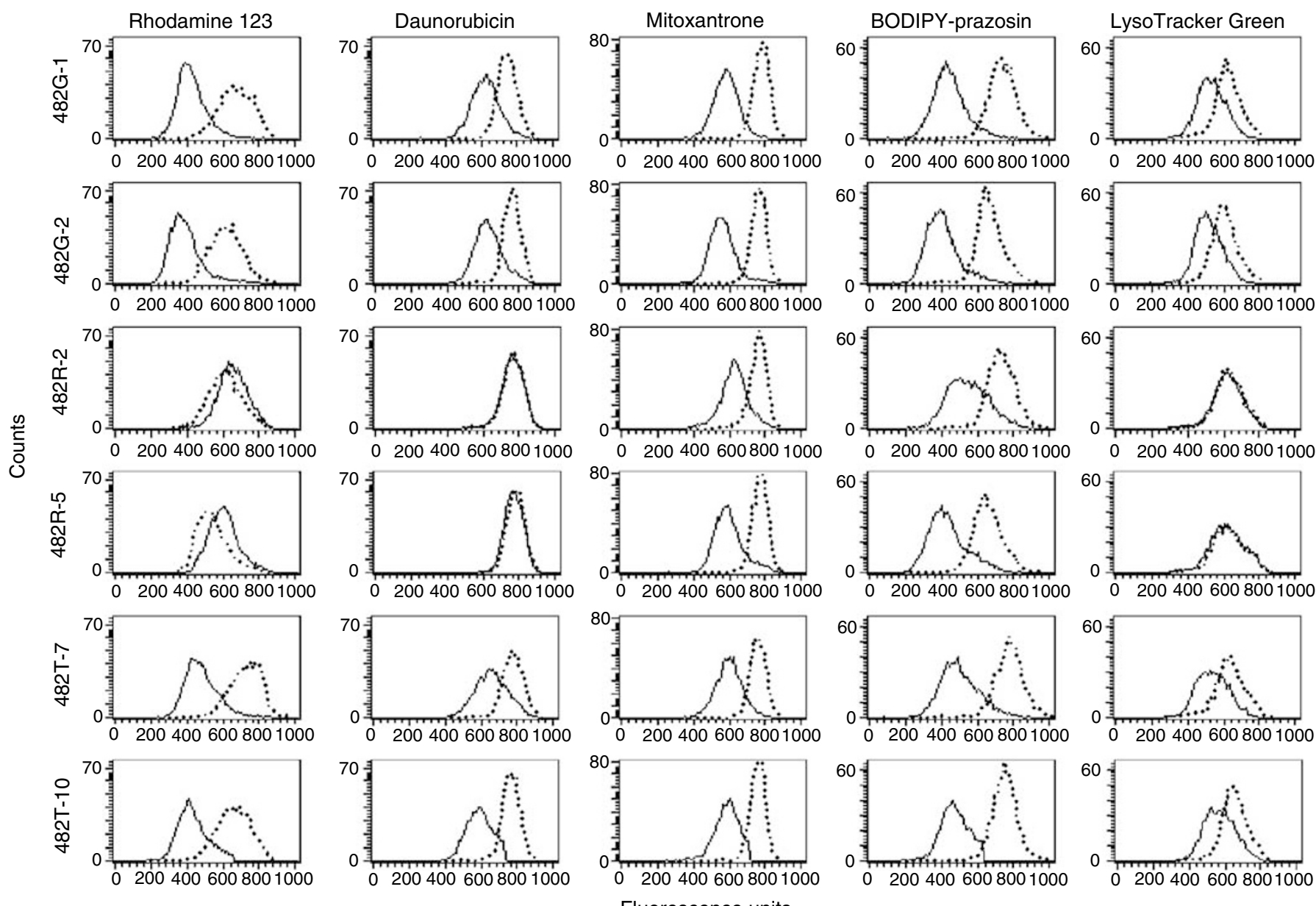

Figure 2 Transport of fluorescent substrates by wild-type and mutant ABCG2. The six transfected cell lines were incubated in rhodamine 123 $\left(0.5 \mu \mathrm{g} \mathrm{ml}^{-1}\right)$, daunorubicin $\left(5 \mu \mathrm{g} \mathrm{ml^{-1 }}\right)$, mitoxantrone $(20 \mu \mathrm{M})$, BODIPY-prazosin $(250 \mathrm{nM})$, or LysoTracker Green $(250 \mathrm{nM})$ with or without I0 $\mu \mathrm{M}$ FTC for 30 min at $37^{\circ} \mathrm{C}$. Cells were then washed, allowed to efflux for I h at $37^{\circ} \mathrm{C}$ in substrate-free media continuing with (dotted line) or without (solid line) FTC, and analysed on a flow cytometer. Representative results are shown. Mean channel differences from 2 or more experiments are present in Table 1.

obtained from cells incubated in $10 \mu \mathrm{M}$ FTC was considered maximal accumulation. The values in Table 3 were generated by subtracting the mean channel number of the inhibitor/efflux histogram from the FTC/efflux histogram. The more effective the inhibitor, the smaller the difference between the inhibitor/efflux histogram and the FTC/efflux histogram. Values in the efflux column were obtained by subtracting the mean channel value of the efflux histogram from the FTC/efflux histogram, providing a measure of ABCG2 activity in each cell line. Both of the $482 \mathrm{R}$ clones show slightly lower BODIPY-prazosin efflux than the mutant clones, a result that parallels the mitoxantrone cross- resistance and transport data. The compounds $\beta$-estradiol and estrone (Imai et al, 2002) have also recently been reported to inhibit ABCG2-mediated transport. We confirm that both compounds do block ABCG2, although $\beta$-estradiol appears to be more effective than estrone.

The ability of the putative ABCG2 antagonist novobiocin (Doyle et al, 2002; Shiozawa et al, 2002) to block ABCG2-mediated efflux of BODIPY-prazosin was most affected by the amino acid at position 482 . The difference between the $50 \mu \mathrm{m}$ novobiocin/efflux histogram and the FTC/efflux histogram was smallest for cells transfected with wild-type ABCG2, suggesting that novobiocin is 
more effective in inhibiting wild-type-mediated transport, as shown in Table 3. Efflux mediated by the mutant ABCG2 proteins was nearly unaffected by novobiocin. Increasing the concentration of novobiocin to $100 \mu \mathrm{m}$ almost completely abrogated wild-type ABCG2-mediated efflux. Histograms for the transfectants are shown in Figure 4A. BODIPY-prazosin fluorescence in the presence of $50 \mu \mathrm{M}$ novobiocin (dotted line) in HEK-293 cells transfected with wild-type ABCG2 was comparable to that in cells incubated with $10 \mu \mathrm{M}$ FTC (dashed line). For 482R-5, these histograms overlap. However, novobiocin was less effective in mutant $482 \mathrm{G}$ and $482 \mathrm{~T}$ ABCG2, where BODIPY-prazosin accumulation with novobiocin is near that of cells incubated with BODIPY-prazosin alone (solid line).

To verify the flow cytometry results, 4-day cytotoxicity assays were then performed on HEK-293 cells transfected with wild-type and mutant ABCG2 in the presence of topotecan with (filled symbols) and without (open symbols) $50 \mu \mathrm{m}$ novobiocin as shown in Figure 4B. Topotecan was selected because cross-resistance studies (in Table 2) suggested that cells transfected with wild-type ABCG2 exhibited slightly higher resistance to the drug compared to the mutant proteins. In empty vector transfected cells (squares) as well as the mutant $482 \mathrm{G}-1$ and $482 \mathrm{~T}-10$ cells (circles), $50 \mu \mathrm{M}$ novobiocin had little effect on topotecan cytotoxicity. Cells transfected with 482G ABCG2 were 21-fold resistant to topotecan (filled circles) and 14 -fold resistant in the presence of $50 \mu \mathrm{M}$ novobiocin (open circles), while cells transfected with $482 \mathrm{~T}$ ABCG2 were 29-fold resistant to topotecan (filled circles) and were nine-fold resistant to the drug in the presence of novobiocin (open circles). In contrast, HEK-293 cells transfected with wild-type ABCG2 (482R) were 71-fold resistant to topotecan (filled circles), and were only three-fold resistant to the drug in the presence of $50 \mu \mathrm{m}$ novobiocin. Thus, novobiocin nearly completely reversed wild-type ABCG2-mediated resistance to topotecan. Dose-modifying factors (DMFs) were also calculated for each transfectant by dividing the $\mathrm{IC}_{50}$ for topotecan alone by the $\mathrm{IC}_{50}$ of topotecan in the presence of novobiocin and are given in Figure 4B.

\section{DISCUSSION}

To confirm earlier transport and resistance studies conducted with selected cell lines overexpressing wild-type and mutant ABCG2 (Honjo et al, 2001; Robey et al, 2001a), HEK-293 cells were transfected with vectors encoding wild-type or mutant ABCG2. Our results with these transfectants confirm that the fluorescent compounds mitoxantrone, BODIPY-prazosin, and Hoechst 33342 are substrates of all three ABCG2 proteins tested in the present study, while LysoTracker Green, daunorubicin, and rhodamine 123 are only appreciably transported by the mutant $482 \mathrm{G}$ and $482 \mathrm{~T}$ proteins. In cytotoxicity assays, all ABCG2 proteins conferred high levels of resistance to mitoxantrone, topotecan, and SN-38, while the mutant proteins conferred four times more resistance to mitoxantrone than the wild-type protein. Cells transfected with wild-type ABCG2 appeared slightly more resistant to SN-38 and topotecan. The mutant $482 \mathrm{G}$ and $482 \mathrm{~T}$ ABCG2 proteins conferred
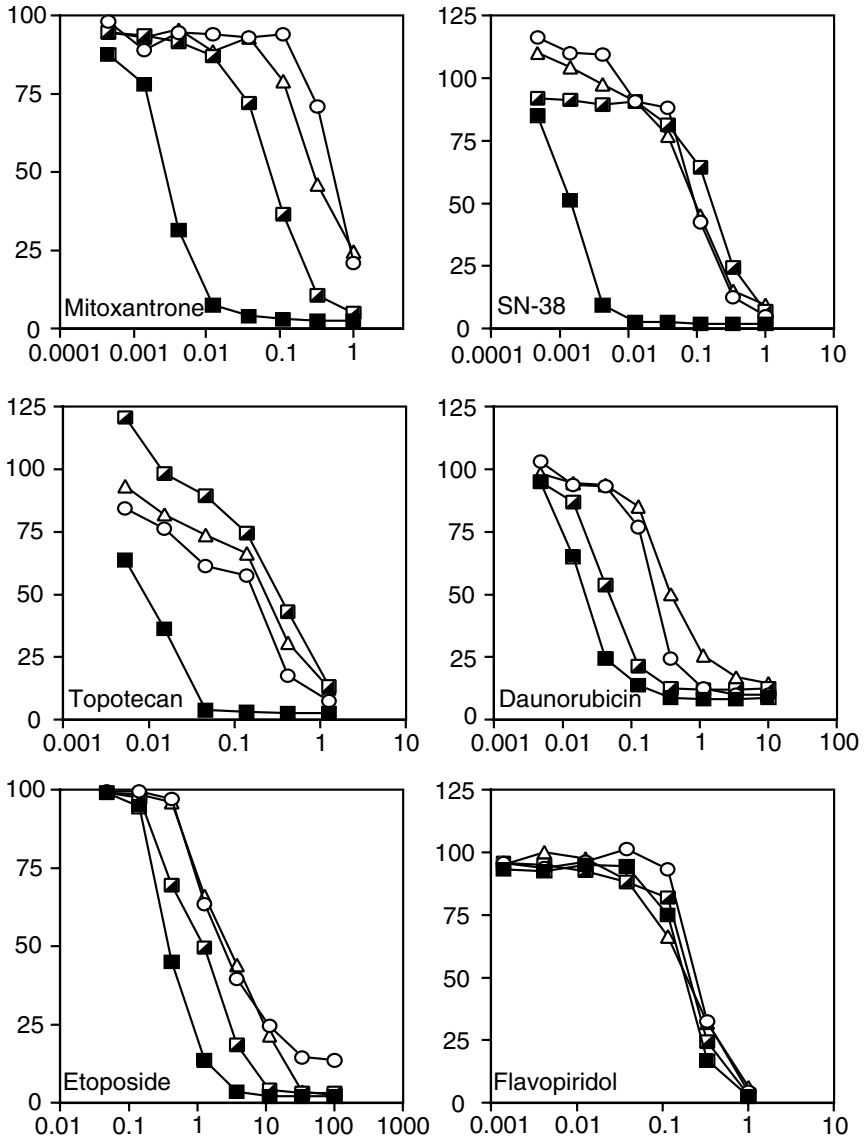

Figure 3 Cross-resistance profile conferred by wild-type and mutant ABCG2. The 4-day cytotoxicity assays were performed with mitoxantrone, SN-38, topotecan, daunorubicin, etoposide, and flavopiridol on HEK-293 cells transfected with empty vector (filled squares), or transfected with 482R (hatched squares), 482G (open circles), or 482T (open triangles) ABCG2. Curves representative of two to five separate experiments are shown.

Table 2 Cross-resistance profile of HEK-293 cells transfected with wild-type and mutant ABCG2

\begin{tabular}{|c|c|c|c|c|c|c|c|}
\hline \multirow[b]{2}{*}{ Drug } & \multirow{2}{*}{$\begin{array}{l}\text { pcDNA3 } \\
\text { IC50 (nM) }\end{array}$} & \multicolumn{2}{|c|}{ 482G-I } & \multicolumn{2}{|c|}{ 482R-2 } & \multicolumn{2}{|c|}{ 482T- 10} \\
\hline & & IC50 (nM) & $\mathbf{R R}^{\mathbf{a}}$ & IC50 (nM) & $\mathbf{R R}$ & IC50 (nM) & $\mathbf{R R}$ \\
\hline Mitoxantrone & $3.8 \pm 1.8$ & $543 \pm 53$ & 140 & $107 \pm 64$ & 28 & $457 \pm 287$ & 118 \\
\hline Topotecan & $8 \pm 2$ & $175 \pm 50$ & 22 & $275 \pm 50$ & 34 & $167 \pm 65$ & 21 \\
\hline SN-38 & $1.2 \pm 1.1$ & $65 \pm 35$ & 52 & $120 \pm 113$ & 96 & $60 \pm 42$ & 48 \\
\hline Doxorubicin & $11.2 \pm 6.3$ & $575 \pm 171$ & 51 & $35 \pm 13$ & 3 & $800 \pm 245$ & 71 \\
\hline Daunorubicin & $20 \pm 0.1$ & $200 \pm 1$ & 10 & $40 \pm 1$ & 2 & $333 \pm 58$ & 17 \\
\hline Bisantrene & $127 \pm 64$ & $1667 \pm 577$ & 13 & $633 \pm 115$ & 5 & $1667 \pm 577$ & 13 \\
\hline Epirubicin & $25 \pm 7.1$ & $600 \pm|4|$ & 24 & $95 \pm 7.1$ & 4 & $700 \pm 424$ & 28 \\
\hline Paclitaxel & $9 \pm 1$ & $20 \pm 1$ & 2 & $30 \pm 14$ & 3 & $30 \pm 1$ & 3 \\
\hline Depsipeptide & $4 \pm 1$ & $6 \pm 1$ & 1.5 & $8 \pm 1$ & 2 & $7.5 \pm 1$ & 2 \\
\hline Flavopiridol & $123 \pm 68$ & $200 \pm 10$ & 2 & $160 \pm 69$ & I & $200 \pm 10$ & 2 \\
\hline Etoposide & $75 \pm 7$ & $650 \pm 71$ & 9 & $250 \pm 7 \mid$ & 3 & $450 \pm 212$ & 6 \\
\hline Rhodamine 123 & $7000 \pm 2000$ & $267000 \pm 115000$ & 38 & $53000 \pm 12000$ & 8 & $267000 \pm 58000$ & 38 \\
\hline
\end{tabular}

${ }^{a}$ Relative resistance values were obtained by dividing the $I C_{50}$ value of the $A B C G 2$-transfected cell lines by the $I C_{50}$ value of the empty vector transfected cell line. Values obtained were from two to five separate experiments. 
10- to 71-fold resistance to the Pgp substrates doxorubicin daunorubicin, and epirubicin. In contrast, wild-type 482R ABCG2 conferred only three- to four-fold resistance to these agents. Thus, while flow cytometric analysis suggests no transport of daunorubicin or rhodamine 123 in cells transfected with wild-type ABCG2, cross-resistance studies do suggest that wild-type ABCG2 confers a very low level of resistance to these two compounds. All three ABCG2 proteins conferred low levels of resistance to etoposide, while no appreciable resistance to paclitaxel, depsipeptide or flavopiridol was noted.

Mutations at amino-acid 482 have included R482G and R482T in human cancer cells; R482S and R482M in mouse fibroblast lines (Honjo et al, 2001; Allen et al, 2002); and a recently reported R482M mutation in a doxorubicin-selected human T-cell line (Wang et al, 2003). Since amino-acid 482 is predicted to be at the beginning of the third transmembrane segment on the intracellular surface of the membrane, it could be directly involved in substrate binding or could affect substrate transport through structural alterations in the third transmembrane segment (Honjo et al, 2001). Although Allen et al (2002) have suggested that loss of the basic arginine at amino-acid 482 may be critical to substrate specificity, mechanistic studies are needed to understand the changes that result from alteration at aminoacid 482.

Since the wild-type arginine is a bulky, positively charged amino acid, it could impede binding or transport of positively charged compounds. While this could explain the poor transport of doxorubicin and daunorubicin, both of which possess aliphatic amino groups that are positively charged at a physiological $\mathrm{pH}$ (7.6), we would note that mitoxantrone, a substrate for all ABCG2 proteins, has a similar structure and charge. LysoTracker and BODIPY-prazosin both carry a positive charge on a boron atom, thus resembling rhodamine's positively charged amino group, yet only prazosin is a substrate for all three ABCG2 proteins. These observations suggest that a positive charge alone is not predictive of whether a compound will be an ABCG2 substrate.

Table 3 Inhibition of BODIPY-prazosin efflux in ABCG2 transfectants assayed by flowcytometry

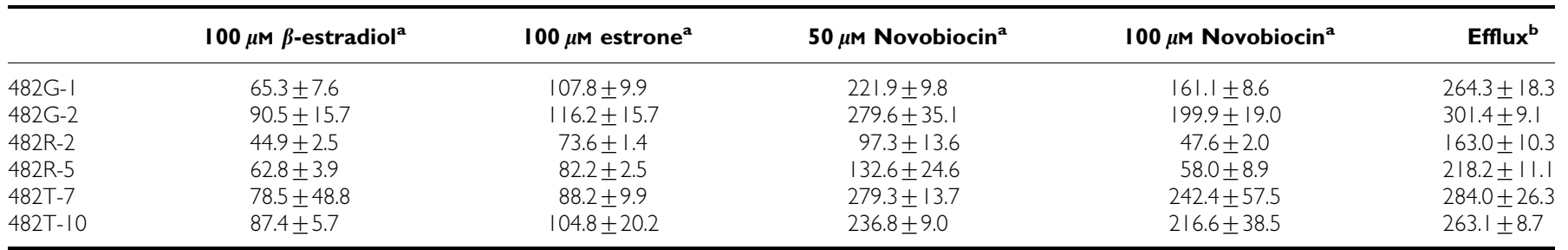

a The values shown are the difference in mean channel numbers between the FTC/efflux histogram and the inhibitor/efflux histogram of the noted inhibitor. ${ }^{b}$ Difference in mean channel number between the FTC/efflux histogram and the efflux histogram. Values were obtained from two separate experiments.
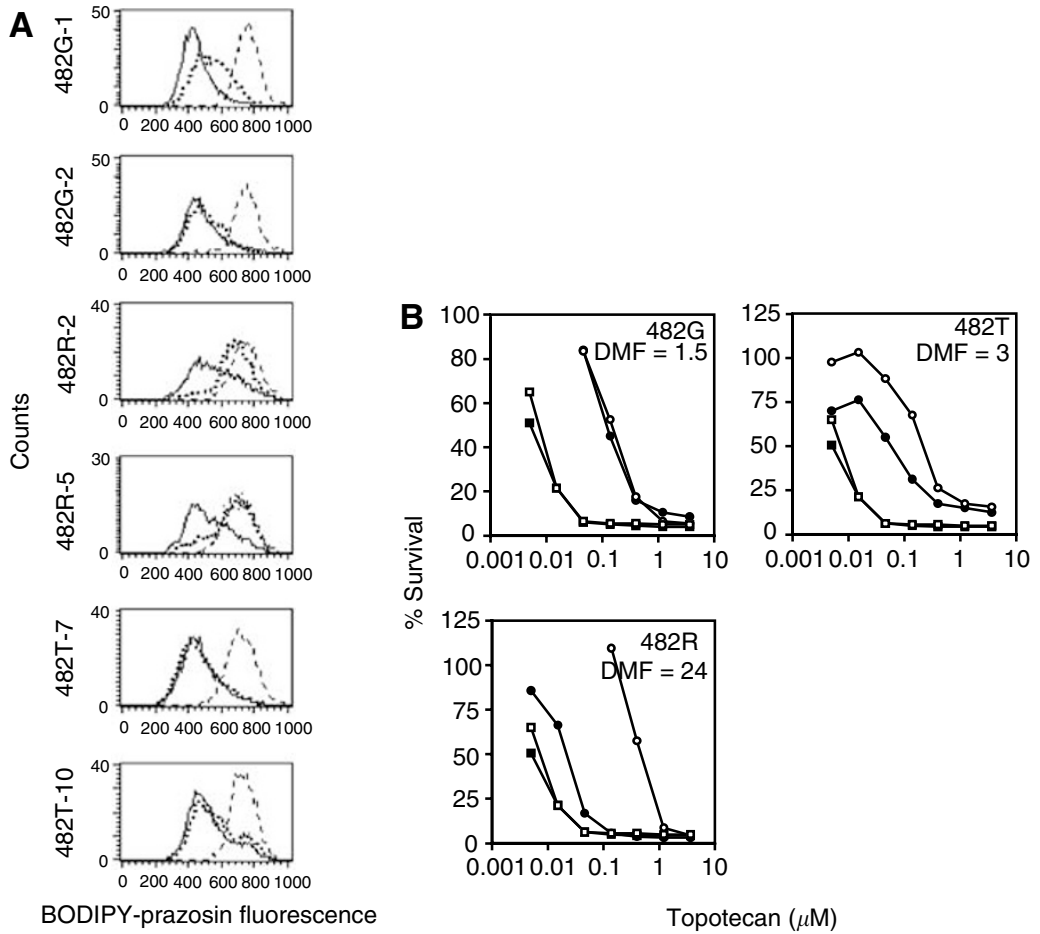

Figure 4 Novobiocin reverses wild-type ABCG2 but not mutant ABCG2-mediated resistance. (A) The six transfected cell lines were incubated in 250 nM BODIPY-prazosin with or without $50 \mu \mathrm{M}$ novobiocin for $30 \mathrm{~min}$ at $37^{\circ} \mathrm{C}$, washed, then allowed to efflux for I h at $37^{\circ} \mathrm{C}$ continuing with (dotted line) or without (solid line) novobiocin and compared to cells incubated in $10 \mu \mathrm{M}$ FTC (dashed line). (B) The 4-day cytotoxicity assays were performed with topotecan with (filled symbols) or without (open symbols) $50 \mu \mathrm{M}$ novobiocin on HEK-293 cells expressing wild-type (482R) or mutant (482G, 482T) ABCG2 as detailed in the Materials and Methods section. Representative results are shown. Empty vector transfected cells are shown as squares while ABCG2 transfected cells are denoted by circles. Dose-modifying factor values were obtained for the ABCG2-transfected cells by dividing the IC ${ }_{50}$ for topotecan without novobiocin by the $\mathrm{IC}_{50}$ value for topotecan in the presence of novobiocin. 
Resistance to the anionic methotrexate has also recently been shown to be affected by mutations in ABCG2 at amino-acid 482 . Volk et al report that selected cell lines expressing wild-type ABCG2 are resistant to methotrexate. Loss of the positive charge at aminoacid 482 by mutation to glycine or threonine yields a protein conferring much less resistance to the drug (Volk et al, 2002). It seems reasonable to assume that mutations at amino-acid 482 in ABCG 2 could affect the ability of the protein to confer resistance to other drugs currently being evaluated for use in the clinic.

The findings presented here parallel those of Allen et al, (2002), who described two mutations, R482M and R482S, in mouse fibroblast cells lacking functional Mdr1, Mdr2, and Mrp1. These authors observed greater anthracycline resistance, lower topotecan resistance and enhanced transport of rhodamine 123. Unlike our observations in human cells where a change from arginine to glycine or threonine occurred early in the course of the drug selection, in the mouse fibroblasts selected with doxorubicin, overexpression and amplification of wild-type Abcg2 occurred before mutation at amino-acid 482. This would suggest that murine Abcg2 can confer biologically meaningful resistance to doxorubicin; a finding that appears to be in conflict with the very low levels of resistance we observed in HEK-293 cells transfected with wild-type human ABCG2. This may simply be due to differing substrate affinities in the ABCG2 protein in the two species, as has been reported for MRP1 (Stride et al, 1997).

Mutations at amino-acid 482 also were found to alter the efficacy of a reported ABCG2 inhibitor. Novobiocin, a compound recently reported to block ABCG2-mediated efflux (Doyle et al, 2002; Shiozawa et al, 2002), was found to be most effective on wild-type ABCG2 and nearly ineffective on mutant ABCG2. This would suggest that, if the described R482T or R482G mutations in ABCG2 were to occur in patients, they could render currently known ABCG2 inhibitors less effective. FTC at $10 \mu \mathrm{M}$ appeared to inhibit all of the ABCG2 proteins equally well. Both $\beta$-estradiol and estrone inhibited ABCG2-mediated prazosin transport; however, estrone was the least effective of the two.

\section{REFERENCES}

Allen JD, Brinkhuis RF, Wijnholds J, Schinkel AH (1999) The mouse Bcrp1/ Mxr/Abcp gene: amplification and overexpression in cell lines selected for resistance to topotecan, mitoxantrone, or doxorubicin. Cancer Res 59: $4237-4241$

Allen JD, Jackson SC, Schinkel AH (2002) A mutation hot spot in the Bcrp1 (Abcg2) multidrug transporter in mouse cell lines selected for doxorubicin resistance. Cancer Res 62: 2294-2299

Allikmets R, Schriml LM, Hutchinson A, Romano-Spica V, Dean M (1998) A human placenta-specific ATP-binding cassette gene (ABCP) on chromosome $4 \mathrm{q} 22$ that is involved in multidrug resistance. Cancer Res 58: $5337-5339$

Brangi M, Litman T, Ciotti M, Nishiyama K, Kohlhagen G, Takimoto C, Robey R, Pommier Y, Fojo T, Bates SE (1999) Camptothecin resistance: role of the ATP-binding cassette $(\mathrm{ABC})$, mitoxantrone-resistance halftransporter (MXR), and potential for glucuronidation in MXR-expressing cells. Cancer Res 59: 5938-5946

Chen G, Duran GE, Steger KA, Lacayo NJ, Jaffrezou JP, Dumontet C, Sikic BI (1997) Multidrug-resistant human sarcoma cells with a mutant Pglycoprotein altered phenotype and resistance to cyclosporins. $J$ Biol Chem 272: $5974-5982$

Chen Y-N, Mickley LA, Schwartz AM, Acton EM, Hwang J, Fojo AT (1990) Characterization of adriamycin-resistant human breast cancer cells which display overexpression of a novel resistance-related membrane protein. J Biol Chem 265: $10073-10080$

Doyle LA, Wei D, Yang W, Nakanishi T, Ross DD (2002) Modulation of mitoxantrone daunorubicin topotecan and flavopiridol cytotoxicity in BCRP-expressing cancer cells by novobiocin. Proc Am Assoc Cancer Res 43: 495 - 496 (abstract)

Doyle LA, Yang W, Abruzzo LV, Krogmann T, Gao Y, Rishi AK, Ross DD (1998) A multidrug resistance transporter from human MCF-7 breast cancer cells. Proc Natl Acad Sci USA 95: 15665-15670
It was surprising that the transfected cells displayed no resistance to flavopiridol, since we have reported overexpression of ABCG2 in cells selected with flavopiridol and have shown crossresistance to flavopiridol in ABCG2 overexpressing selected cell lines (Robey et al, 2001b). This could be due to the fact that flavopiridol is a substrate with a weak affinity to ABCG2 and thus the expression of ABCG2 in the transfected cells is inadequate to confer appreciable resistance. In support of this explanation is the cross-resistance data obtained in a cell line selected in flavopiridol (MCF-7 FLV1000) which was only 24-fold resistant to flavopiridol but was 675 -fold resistant to mitoxantrone. Alternatively, modification of flavopiridol may be necessary to allow it to be better transported. Recent studies have shown that flavopiridol is most likely glucuronidated in human liver by UDP-glucuronosyltransferase isoforms (Ramirez et al, 2002). We have previously suggested a link between glucuronidation and resistance mediated by ABCG2, as we noted increased expression of UDP-glucuronosyltransferase in cell lines that overexpress ABCG2 (Brangi et al, 1999). It is thus possible that breast carcinoma cells selected in flavopiridol have the capacity to glucuronidate flavopiridol, while HEK-293 cells do not. Studies are currently underway to determine if flavopiridol-selected cells do indeed glucuronidate flavopiridol in order to transport it.

In summary, we show that mutation of a single amino acid in the ABCG2 protein has a major effect on its substrate specificity, and may possibly undermine the effectiveness of potential ABCG2 blockers.

\section{ACKNOWLEDGEMENTS}

We thank Bill Telford and Veena Kapoor of the National Cancer Institute Flow Cytometry Unit for help with flow cytometry analyses. We also thank Michael Rapawy and Robert Swendiman for their excellent technical assistance, and Zhirong Zhan for cell culture work. We also appreciate the help of Ven Narayanan.

Honjo Y, Hrycyna CA, Yan QW, Medina-Perez WY, Robey RW, van de Laar A, Litman T, Dean M, Bates SE (2001) Acquired mutations in the MXR/ $\mathrm{BCRP} / \mathrm{ABCP}$ gene alter substrate specificity in MXR/BCRP/ABCP-overexpressing cells. Cancer Res 61: 6635-6639

Imai Y, Tsukahara S, Ishikawa E, Tsuruo T, Sugimoto Y (2002) Estrone and 17beta-estradiol reverse breast cancer resistance protein-mediated multidrug resistance. Jpn J Cancer Res 93: 231 - 235

Kawabata S, Oka M, Shiozawa K, Tsukamoto K, Nakatomi K, Soda H, Fukuda M, Ikegami Y, Sugahara K, Yamada Y, Kamihira S, Doyle LA, Ross DD, Kohno S (2001) Breast cancer resistance protein directly confers SN-38 resistance of lung cancer cells. Biochem Biophys Res Commun 280: $1216-1223$

Kim M, Turnquist H, Jackson J, Sgagias M, Yan Y, Gong M, Dean M, Sharp JG, Cowan K (2002) The multidrug resistance transporter ABCG2 (breast cancer resistance protein 1) effluxes Hoechst 33342 and is overexpressed in hematopoietic stem cells. Clin Cancer Res 8: 22-28

Litman T, Brangi M, Hudson E, Fetsch P, Abati A, Ross DD, Miyake K, Resau JH, Bates SE (2000) The multidrug-resistant phenotype associated with overexpression of the new ABC half-transporter, MXR (ABCG2). J Cell Sci 113: $2011-2021$

Ma JF, Grant G, Melera PW (1997) Mutations in the sixth transmembrane domain of P-glycoprotein that alter the pattern of cross-resistance also alter sensitivity to cyclosporin A reversal. Mol Pharmacol 51: 922- 930

Maliepaard M, Scheffer GL, Faneyte IF, van Gastelen MA, Pijnenborg AC, Schinkel AH, van De Vijver MJ, Scheper RJ, Schellens JH (2001) Subcellular localization and distribution of the breast cancer resistance protein transporter in normal human tissues. Cancer Res 61: $3458-3464$

Maliepaard M, van Gastelen MA, de Jong LA, Pluim D, van Waardenburg RC, Ruevekamp-Helmers MC, Floot BG, Schellens JH (1999) Over- 
expression of the BCRP/MXR/ABCP gene in a topotecan-selected ovarian tumor cell line. Cancer Res 59: 4559-4563

Miyake K, Mickley L, Litman T, Zhan Z, Robey R, Cristensen B, Brangi M, Greenberger L, Dean M, Fojo T, Bates SE (1999) Molecular cloning of cDNAs which are highly overexpressed in mitoxantrone-resistan cells: demonstration of homology to $\mathrm{ABC}$ transport genes. Cancer Res 59: $8-13$

Rabindran SK, He H, Singh M, Brown E, Collins KI, Annable T, Greenberger LM (1998) Reversal of a novel multidrug resistance mechanism in human colon carcinoma cells by fumitremorgin C. Cancer Res 58: $5850-5858$

Rabindran SK, Ross DD, Doyle LA, Yang W, Greenberger LM (2000) Fumitremorgin $\mathrm{C}$ reverses multidrug resistance in cells transfected with the breast cancer resistance protein. Cancer Res 60: 47-50

Ramirez J, Iyer L, Journault K, Belanger P, Innocenti F, Ratain MJ, Guillemette C (2002) In vitro characterization of hepatic flavopiridol metabolism using human liver microsomes and recombinant UGT enzymes. Pharm Res 19: 588-594

Robey RW, Honjo Y, van de Laar A, Miyake K, Regis JT, Litman T, Bates SE (2001a) A functional assay for detection of the mitoxantrone resistance protein MXR (ABCG2). Biochim Biophys Acta 1512: $171-182$

Robey RW, Medina-Perez WY, Nishiyama K, Lahusen T, Miyake K, Litman T, Senderowicz AM, Ross DD, Bates SE (2001b) Overexpression of the ATP-binding cassette half-transporter ABCG2 (MXR/BCRP/ABCP1), in flavopiridol-resistant human breast cancer cells. Clin Cancer Res 7: $145-152$

Scharenberg CW, Harkey MA, Torok-Storb B (2002) The ABCG2 transporter is an efficient Hoechst 33342 efflux pump and is preferentially expressed by immature human hematopoietic progenitors. Blood 99: 507-512
Shiozawa K, Oka M, Nakatomi K, Yoshikawa M, Ikegama Y, Kawabata S, Soda H, Tsurutani J, Murase K, Kohno S (2002) Effective reversal of breast cancer resistant protein (BCRP)-mediated drug resistance by novobiocin. Proc Am Assoc Cancer Res 43: 778 (abstract)

Skehan P, Stornet R, Scudiero D, Monks A, McMahon J, Vistica D, Warren J, Bokbosch H, Kenny S, Boyd M (1990) New colorimetric cytotoxicity assay for anticancer-drug screening. J Natl Cancer Inst $\mathbf{8 2}$ $1107-1112$

Stride BD, Grant CE, Loe DW, Hipfner DR, Cole SP, Deeley RG (1997) Pharmacological characterization of the murine and human orthologs of multidrug-resistance protein in transfected human embryonic kidney cells. Mol Pharmacol 52: 344-353

Volk EL, Farley KM, Wu Y, Li F, Robey RW, Schneider E (2002) Overexpression of wild-type breast cancer resistance protein mediates methotrexate resistance. Cancer Res 62: $5035-5040$

Wang X, Furukawa T, Nitanda T, Okamoto M, Sugimoto Y, Akiyama S, Baba M (2003) Breast cancer resistance protein (BCRP/ABCG2) induces cellular resistance to HIV-1 nucleoside reverse transcriptase inhibitors. Mol Pharmacol 63: 65-72

Watanabe T, Uchiyama N, Roninson IB, Cohen D, Atadja P (2000) Altered activity of MDR-reversing agents on KB3-1 cells transfected with Gly(185) - > Val human P-glycoprotein. Int J Oncol 17: 579-586

Yang CH, Schneider E, Kuo ML, Volk EL, Rocchi E, Chen YC (2000) BCRP/ MXR/ABCP expression in topotecan-resistant human breast carcinoma cells. Biochem Pharmacol 60: 831-837

Zhou S, Schuetz JD, Bunting KD, Colapietro AM, Sampath J, Morris JJ, Lagutina I, Grosveld GC, Osawa M, Nakauchi H, Sorrentino BP (2001) The ABC transporter Bcrp1/ABCG2 is expressed in a wide variety of stem cells and is a molecular determinant of the side-population phenotype. Nat Med 7: 1028 - 1034 